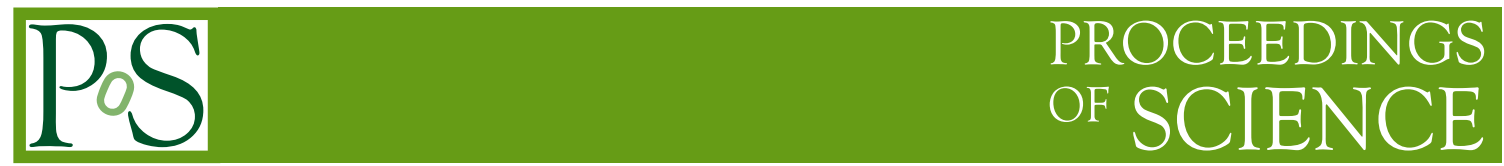

\title{
Irradiation setup at the U-120M cyclotron facility
}

\section{Isakov Artem;; Filip Krizek; Tomas Matlocha}

Nuclear Physics Institute of the Czech Academy of Sciences, Rez, Czechia

Faculty of Nuclear Sciences and Physical Engineering, Czech Technical University

E-mail: isakov@ujf.cas.cz

We present a setup that is used for tests of radiation hardness at the U-120M cyclotron facility at the Nuclear Physics Institute of the Czech Academy of Sciences (NPI CAS). Methods which are used for on-line monitoring of beam parameters and total ionizing dose are discussed. The facility is extensively used for tests of radiation hardness of various electronic components for high-energy physics experiments including silicon sensors and FPGAs.

International Conference on High Energy Physics - ICHEP2020-

28 July - 6 August, 2020

Prague, Czech Republic

*Speaker. 
Electronic components of high-energy physics detectors are often exposed to intensive fluxes of ionization radiation. This radiation deteriorates properties of materials and can introduce false signals in complex electronic circuits. Successful operation of these detectors thus requires to perform tests which show that all sensitive components are radiation hard enough.

The setup for radiation hardness studies, described in this proceeding, uses a $30 \mathrm{MeV}$ proton beam provided by the isochronous cyclotron U-120M of the NPI CAS in Rez [1]. The cyclotron can be operated in positive and negative modes. In the positive mode, the cyclotron accelerates positive ions $\mathrm{H}^{+}, \mathrm{D}^{+},{ }^{3} \mathrm{He}^{2+}$, and ${ }^{4} \mathrm{He}^{2+}$. They are extracted from the cyclotron by means of a three section electrostatic deflection system with a magnetic kicker. In the negative mode, the cyclotron can accelerate negative ions $\mathrm{H}^{-}$and $\mathrm{D}^{-}$. The output beam is extracted by an $1 \mu \mathrm{m}$ thick carbon stripping foil. A negative ion loses both valence electrons by passing through the foil and the positively charged ion is directed by a magnetic field to a beamline. The energies of the extracted proton beam varies in the range from 6 to $37 \mathrm{MeV}$ with the maximal current up to $50 \mu \mathrm{A}$. Tests of radiation hardness are carried out with the negative mode proton beam.

The beam current is controlled via modulation by a $150 \mathrm{~Hz}$ macropulsed signal. A duty cycle of the macropulse signal determines the frequency of proton microbunches injection to the cyclotron and can be adjusted in the range from 5 to $65 \%$. The microbuch structure of the beam is given by the cyclotron radiofrequency which is tunable from $10 \mathrm{MHz}$ to $26 \mathrm{MHz}$ [3].

A simplified scheme of the experimental setup which is used for radiation hardness tests is shown in Fig. 1. The end of the beamline is equipped with an energy degrader unit which allows for insertion of aluminum plates with different thickness to the beam. The plates can be used to absorb the proton beam or to change its parameters like energy or width, see Fig. 2.

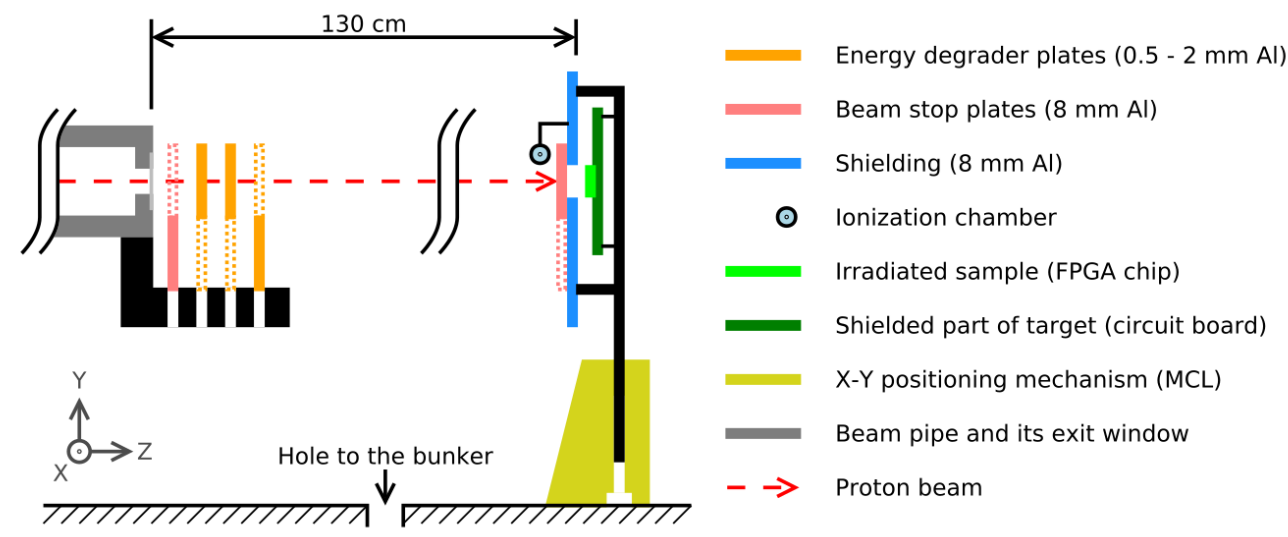

Figure 1: Scheme of the irradiation setup

The irradiated sample is placed on a remotely controlled X-Y positioning mechanism which is located $130 \mathrm{~cm}$ from the beamline. The arm of the setup with the tested sample is movable in transverse plane w.r.t. the beam. The arm further carries another remotely controlled beam shutter to shield the sample, and an ionization chamber which is fixed next to the tested sample and which is used for online monitoring of the proton flux. The chamber provides a linear response to the incoming proton flux up to $10^{9}$ proton $\mathrm{cm}^{-2} \mathrm{~s}^{-1}$ [1].

During irradiation, sample is placed to the beam center and the ionization chamber measures beam intensity at the beam periphery. When estimating the proton flux experienced by the sample, 


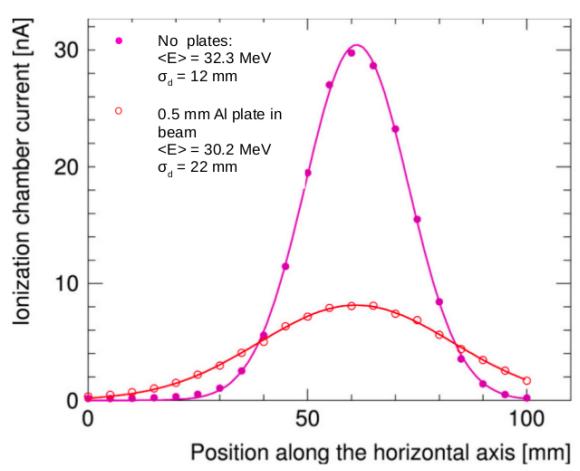

Figure 2: Profile of the proton beam for two energy degrader configurations as measured along the horizontal axis.. The beam profiles measured along the vertical axis look similar. The legend quotes the mean energy of the proton beam at the sample position and the Gaussian width of the beam.

we correct for this displacement by a scaling factor that we know from the measured Gaussian beam profile. The proton flux is monitored each $1 \mathrm{~s}$ and integrated in time to obtain proton fluence. The dose accumulated by the irradiated sample is calculated online using the formula:

$$
D[\mathrm{krad}]=1.602 \times 10^{-8} \times S\left[\mathrm{MeV} \mathrm{cm}^{2} \mathrm{mg}^{-1}\right] \times F\left[\mathrm{~cm}^{-2}\right],
$$

where $S$ is the stopping power of the irradiated material and $F$ is the proton fluence. Estimated systematic uncertainty of absorbed doses is about $10 \%$ [1].

In summary, the presented setup works at the open access irradiation facility CANAM [2] of the NPI CAS and it is suitable to perform radiation hardness studies for various electronic components. The facility was used in several studies, including characterization of FPGAs [4] for the readout unit of the new Inner Tracking System [5] of the ALICE experiment and radiation hardness study of the silicon ALPIDE pixel sensor [6].

Acknowledgements: This research was funded by the Ministry of Education, Youth, and Sports of the Czech Republic, grant number LTT17018.

\section{References}

[1] F. Krizek et al., "Irradiation setup at the U-120M cyclotron facility", Nucl. Instr. Meth. Phys. Res. A 894 (2018) 87-95.

[2] Center of Accelerators and Nuclear Analytical Methods, Nuclear Physics Institute of the CAS: http://canam.ujf.cas.cz/.

[3] M. Cihak, J. Lacman, J. Stursa, "New control system for the isochronous cyclotron U-120M", Proceedings of the 15th Int. Conf. on Cyclotrons and their Applications, Caen, France, 1998, p. 567.

[4] K.M. Sielewicz et al., Prototype readout electronics for the upgraded ALICE Inner Tracking System, JINST 12 (2017) C01008.

[5] B. Abelev et al., (The ALICE Collaboration), "Upgrade of the ALICE experiment: Letter of intent", J. Phys. G: Nucl. Part. Phys. 41 (2014) 087001. 
54

55

[6] S. Kushpil et al., "Recent Results From Beam Tests of the ALPIDE Pixel Chip for the Upgrade of the ALICE Inner Tracker", IEEE TNS 66 (2019) 2319. 